\title{
MicroRNA-200c-3p suppresses intervertebral disc degeneration by targeting RAP2C/ERK signaling
}

\author{
JIANPING CAO $^{1^{*}}$, MENG JIANG $^{2 *}$, HUAFENG REN ${ }^{3}$ and KAI XU ${ }^{2}$ \\ Departments of ${ }^{1}$ Anesthesiology and ${ }^{2}$ Orthopedics, Qingdao No. 6 People's Hospital; ${ }^{3}$ Department of \\ Functional Inspection, Qingdao Haici Medical Treatment Group, Qingdao, Shandong 266033, P.R. China
}

Received May 10, 2021; Accepted August 18, 2021

DOI: $10.3892 / \mathrm{mmr} .2021 .12505$

\begin{abstract}
Intervertebral disc degeneration (IDD) is a major cause of lower back pain. The high morbidity associated with this disease diminishes the quality of life of those who are affected. MicroRNAs (miRs) play crucial roles in various diseases, including IDD. However, the mechanism via which miR-200c-3p plays a role in the development of IDD remains unknown. The present study aimed to investigate the effect of miR-200c-3p on the progression of IDD and the underlying mechanism. The expression level of miR-200c-3p was evaluated in intervertebral disc tissues from patients with IDD. To construct the IDD cell model, the nucleus pulposus (NP) cells were treated with lipopolysaccharide (LPS) $24 \mathrm{~h}$ following transfection with miR-200c-3p mimic or inhibitor. A luciferase activity assay was performed, while reverse transcription-quantitative PCR and western blotting were conducted to determine the RNA and protein expression levels, respectively. The expression level of miR-200c-3p in the intervertebral disc tissues of patients with IDD was lower than that of normal subjects. LPS treatment reduced the expression level of miR-200c-3p in NP cells. Moreover, miR-200c-3p mimic inhibited LPS-induced NP cell apoptosis. It was found that miR-200c-3p attenuated inflammatory cytokine levels and extracellular matrix (ECM) degradation in NP cells. Furthermore, miR-200c-3p targeted Ras-related protein 2C (RAP2C) in NP cells. RAP2C promoted apoptosis, inflammatory cytokine levels and ECM degradation by activating ERK signaling. Knockdown of RAP2C and inhibition of ERK
\end{abstract}

Correspondence to: Dr Kai Xu, Department of Orthopedics, Qingdao No. 6 People's Hospital, 9 Fushun Road, Shibei, Qingdao, Shandong 266033, P.R. China

E-mail: xukai688@163.com

*Contributed equally

Abbreviations: IDD, intervertebral disc degeneration; NP, nucleus pulposus; ECM, extracellular matrix; LPS, lipopolysaccharide; miRNAs/miRs, microRNAs; RAP, Ras-related proteins

Key words: IDD, NP cells, miR-200c-3p, RAP2C, ERK signaling signaling by SCH772984 partially reversed the proinflammatory effect of the miR-200c-3p inhibitor on LPS-treated NP cells. Thus, miR-200c-3p inhibits NP cell apoptosis, inflammatory cytokine levels and ECM degradation in IDD by targeting RAP2C/ERK signaling.

\section{Introduction}

Intervertebral disc degeneration (IDD) is a prevalent complicated disease of the spine that reduces the quality of life of patients (1). IDD is usually associated with myelopathy, radiculopathy and lower back pain, with an incidence rate of $>90 \%$ (2). IDD is affected by several factors, such as apoptosis, inflammatory factors, degradative enzymes, mechanical load, cellular senescence and heredity (3). However, the exact mechanisms responsible for IDD remain elusive.

MicroRNAs (miRNAs/miRs) are small non-coding RNAs that modulate their target genes by regulating mRNA stability and translation, as well as controlling gene expression at the post-transcriptional level by pairing with target mRNAs at the 3 ' untranslated region (3'UTR) (4). Increasing evidence has revealed that miRNAs are involved in the progression of IDD. For instance, miR-140-5p overexpression reduces lipopolysaccharide (LPS)-induced inflammation and degeneration of intervertebral discs by downregulating Toll-like receptor 4 (TLR4) (5). Moreover, miR-132 contributes to extracellular matrix (ECM) degradation by directly targeting growth and differentiation factor 5 (GDF5) in NP cells (6). miR-200c-3p is involved in the pathogenesis of inflammatory diseases (7), and it has been shown that the expression levels of miR-200c-3p in the synovial fluid of 150 patients with knee osteoarthritis were significantly lower compared with those in 54 healthy controls (8). miR-200c-3p has also been reported to inhibit apoptosis and inflammation in cigarette smoke extract-stimulated 16HBE cells (9). However, the mechanism of action of miR-200c-3p on IDD progression remains unknown.

Ras-related proteins (RAPs) organize various biological processes, including metabolic turnover, cytoskeletal organization, cell cycle, differentiation and cell adhesion (10). RAP contains a broad family of nucleotide-binding proteins, including five members: RAP1A, RAP1B, RAP2A, RAP2B and RAP2C (11). Previous studies have revealed that RAP2C was involved in multiple diseases, such as laryngeal squamous cell carcinoma (12). However, to the best of our knowledge, the 
role of RAP2C in the regulation of IDD progression has not been previously reported. It has been shown that RAP2C activates ERK signaling (13), which has been identified as crucial in the modulation of IDD progression $(14,15)$. Pro-inflammatory macrophages promote the degeneration phenotypes of nucleus pulposus (NP) cells partly via JNK and ERK signaling (16). However, the association of ERK signaling with miR-200c-3p and RAP2C in IDD modulation is yet to be fully elucidated.

Thus, the present study aimed to investigate the role and underlying mechanism of miR-200c-3p in the modulation of IDD development.

\section{Materials and methods}

IDD clinical samples. A total of 22 intervertebral disc tissues from patients with IDD (age, 41-64 years; 10 male and 12 female) and 9 normal samples (age, $40-49$ years; 4 male and 5 female) used in this study were obtained from Qingdao No. 6 People's Hospital between May 2018 and December 2019. All patients with chronic lower back pain (lasting for $>3$ months) underwent MRI examinations $(17,18)$. The 22 patients with IDD underwent fusion surgery and intervertebral disc excision. The 9 normal tissues were obtained from patients who underwent traumatic lumbar fracture. Patients who were treated with chemotherapy or radiotherapy were excluded from this study. The samples were immediately frozen in liquid nitrogen and stored at $-80^{\circ} \mathrm{C}$ before further analysis. The samples used in this study were obtained with written approval from patients. This study conformed to the experimental guidelines of the World Medical Association and the Ethics Committee of Qingdao No.6 People's Hospital (approval no. 201907).

Cell culture and treatment. Human NP cells were obtained from ScienCell Research Laboratories, Inc. The cells were cultured in DMEM (Gibco; Thermo Fisher Scientific, Inc.) containing 15\% FBS (Gibco; Thermo Fisher Scientific, Inc.), $0.1 \mathrm{mg} / \mathrm{ml}$ streptomycin (Beijing Solarbio Science \& Technology Co., Ltd.) and $100 \mathrm{U} / \mathrm{ml}$ penicillin (Beijing Solarbio Science \& Technology Co., Ltd.) at $37^{\circ} \mathrm{C}$ with $5 \% \mathrm{CO}_{2}$.

Lentiviral plasmids (GV248) carrying RAP2C short hairpin RNA (shRNA) and the corresponding negative control (NC) shRNA were synthesized and obtained from Shanghai GenePharma Co., Ltd. The sequence of RAP2C shRNA was 5'-GAAGCAAGAUCAGUGUUGU-3' and the sequence of the NC shRNA was 5'-TTCTCCGAACGTGTCACGT-3'. The ratio of the lentiviral plasmid, packaging vector (Shanghai GenePharma Co., Ltd.) and envelope vector (Shanghai GenePharma Co., Ltd.) was 4:3:3. A second-generation system was used. Briefly, 293T cells (American Type Culture Collection) were plated into 6 -well plates ( $1 \times 10^{6} /$ well), which were used as the interim cell line, and they were transfected with $20 \mu \mathrm{g}$ lentiviral plasmid using Lipofectamine ${ }^{\circledR} 3000$ (Invitrogen; Thermo Fisher Scientific, Inc.) at $37^{\circ} \mathrm{C}$ for $12 \mathrm{~h}$. Lentiviral particles were collected using $72,000 \mathrm{x} \mathrm{g} / \mathrm{min}$ centrifugation for $120 \mathrm{~min}$. NP cells were plated into 24-well plates $\left(2 \times 10^{5} /\right.$ well $)$ and subsequently infected with the lentiviral supernatant $\left(1 \times 10^{8} \mathrm{TU} / \mathrm{ml}\right)$ at a multiplicity of infection of 10 at $37^{\circ} \mathrm{C}$ for $12 \mathrm{~h}$ using TR-1003-G (Sigma-Aldrich; Merck $\mathrm{KGaA}$ ). After $48 \mathrm{~h}$ of cell transduction, these cells were selected for in the presence of puromycin $(1 \mu \mathrm{g} / \mathrm{ml}$; Beyotime Institute of
Biotechnology) for 3 days between transfection and experimentation at $37^{\circ} \mathrm{C}$ to generate stable NC and RAP2C-knockdown cells. Puromycin $(0.5 \mu \mathrm{g} / \mathrm{ml})$ was used for maintenance.

pcDNA3.1-RAP2C overexpression vector (OE-RAP2C) and empty pcDNA3.1 plasmid were synthesized and obtained from GenScript. miR-200c-3p mimic, miR-200c-3p inhibitor and corresponding scrambled controls were synthesized and obtained from Shanghai GenePharma Co., Ltd. NP cells (2x10 /well) were transfected with 100 pmol pcDNA-3.1 vectors or $50 \mathrm{nM}$ miRNA constructs using Lipofectamine ${ }^{\circledR}$ 3000 (Invitrogen; Thermo Fisher Scientific, Inc.), according to the manufacturer's instructions, at $37^{\circ} \mathrm{C}$ for $6 \mathrm{~h}$. The sequences of each construct were as follows: miR-200c-3p mimic sense, 5'-UAAUACUGCCGGGUAAUGAUGGA-3' and antisense, 5'-CAUCAUUACCCGGCAGUAUUAUU-3'; mimic NC sense, 5'-UUCUCCGAACGUGUCACGUTT-3' and antisense, 5'-ACGUGACACGUUCGGAGA ATT-3'; miR-200c-3p inhibitor, 5'-UCCAUCAUUACCCGGCAGUAUUA-3'; and inhibitor NC, 5'-CAGUACUUUUGUGUAGUACAA-3'. NP cells were harvested $24 \mathrm{~h}$ after transfection for use in subsequent experiments.

To construct the IDD cell model, NP cells were plated into 6-well plates $\left(3 \times 10^{5} /\right.$ well $)$ and treated with LPS $(1 \mu \mathrm{g} / \mathrm{ml}$; Sigma-Aldrich; Merck KGaA) for $24 \mathrm{~h}$ at $37^{\circ} \mathrm{C}$ after $24 \mathrm{~h}$ of transfection. The ERK inhibitor, SCH772984 (Selleck Chemicals), was used at a dosage of $10 \mu \mathrm{mol} / 1$ for $24 \mathrm{~h}$ at $37^{\circ} \mathrm{C}$.

Reverse transcription-quantitative PCR (RT-qPCR). Total RNAs were extracted using TRIzol ${ }^{\circledR}$ (Invitrogen; Thermo Fisher Scientific, Inc.) and were treated with RQ1 RNase-Free DNase (Promega Corporation) for $30 \mathrm{~min}$ at $37^{\circ} \mathrm{C}$. The quality of total RNA was detected at an absorbance $260 / 280 \mathrm{~nm}$ ratio using NanoDrop life spectrophotometer (Thermo Fisher Scientific, Inc.). cDNA was synthesized with the Maxima First-strand cDNA Synthesis kit according to the manufacturer's instructions (Thermo Fisher Scientific, Inc.). qPCR was performed using the SYBR Real-time PCR I kit (Takara Bio, Inc.). The thermocycling conditions used for the qPCR were as follows: Initial denaturation for $3 \mathrm{~min}$ at $95^{\circ} \mathrm{C}$; followed by 37 cycles of denaturation at $94^{\circ} \mathrm{C}$ for $1 \mathrm{~min}$, annealing at $60^{\circ} \mathrm{C}$ for $1 \mathrm{~min}$ and extension at $72^{\circ} \mathrm{C}$ for $1 \mathrm{~min}$, followed by a final extension step at $72^{\circ} \mathrm{C}$ for $7 \mathrm{~min}$. Relative quantification was performed using the $2^{-\Delta \Delta \mathrm{Cq}}$ method (19). The standard controls for miRNA and mRNA were U6 and GAPDH, respectively (20). Quantitative determination of the RNA levels was performed in three independent experiments. Data are presented as the mean \pm SD. The primer sequences were as follows: miR-200c-3p NCBI Reference Sequences (RefSeq), NC_000012.12; miR-200c-3p forward, 5'-GGGGTAGGGGAAGGTGGTTTA-3' and reverse, 5'-CACCACCCCAATCCCTAA AАACACT-3'; RAP2C NCBI RefSeq, XM_035008753.1; RAP2C forward, 5'-TGGCCATACCGAGCAGATAAAACTCA-3' and reverse, 5'-ACAGGTTTACCAAGGCTCAGTTCTGC-3'; collagen II NCBI RefSeq, XM_008951124.2; collagen II forward, 5'-CTG GTGATGATGGTGAAG-3' and reverse, 5'-CCTGGATAA CCTCTGTGA-3'; AggrecanNCBI RefSeq, NM_001369268.1; aggrecan forward, 5'-GCGAGCACTGTAACATAGACA T-3' and reverse, 5'-TCACACAGGTCCCCTTCGTA-3'; GAPDH NCBI RefSeq, NM_001256799.3; GAPDH forward, 5'-CATGTTGCAACCGGGAAGGA-3' and reverse, 5'-GCC 
A

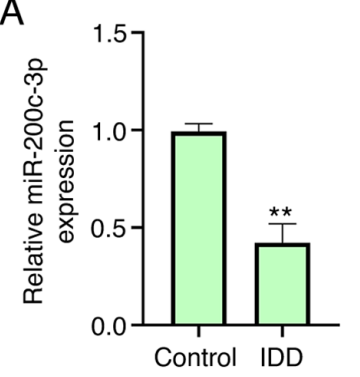

D
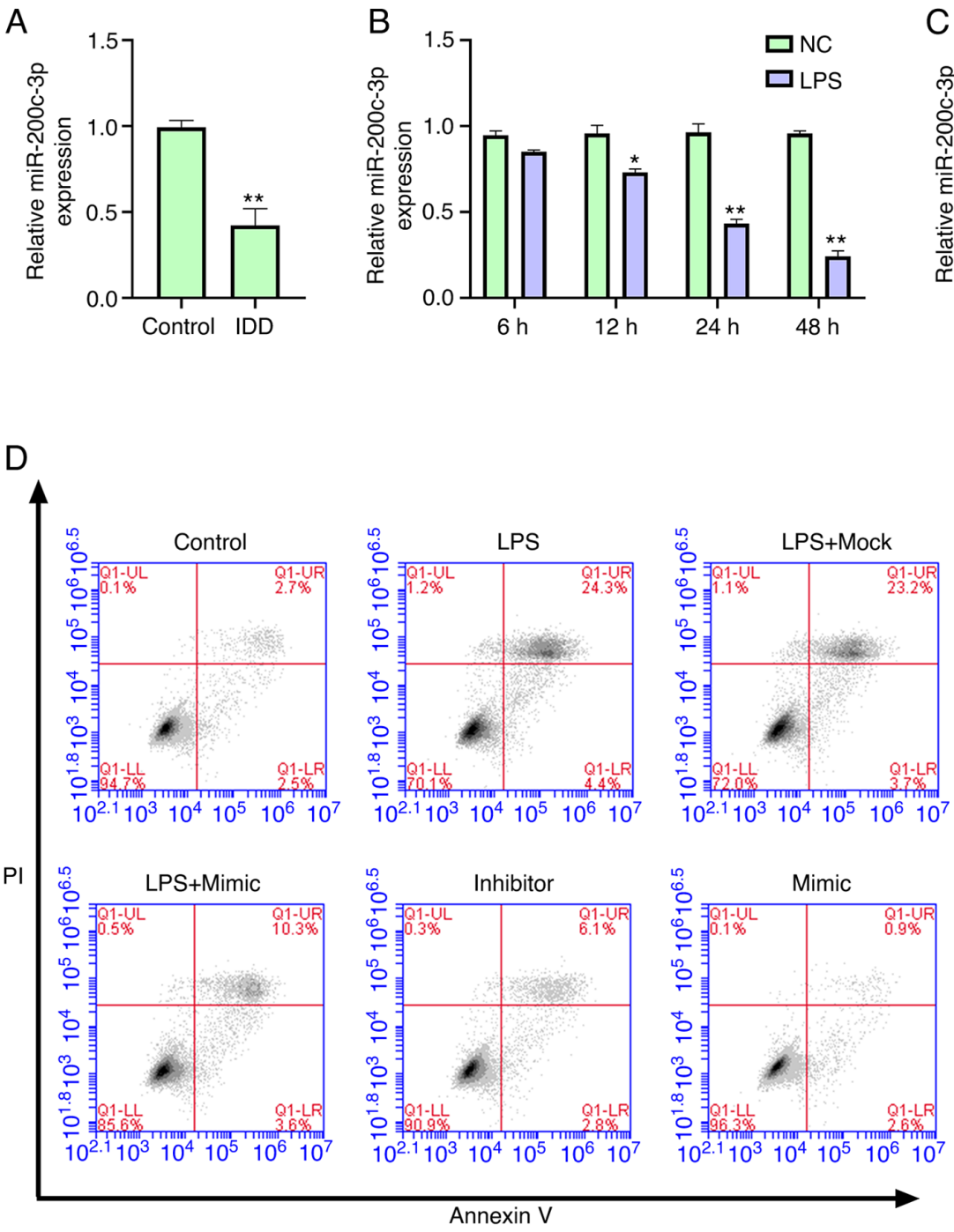
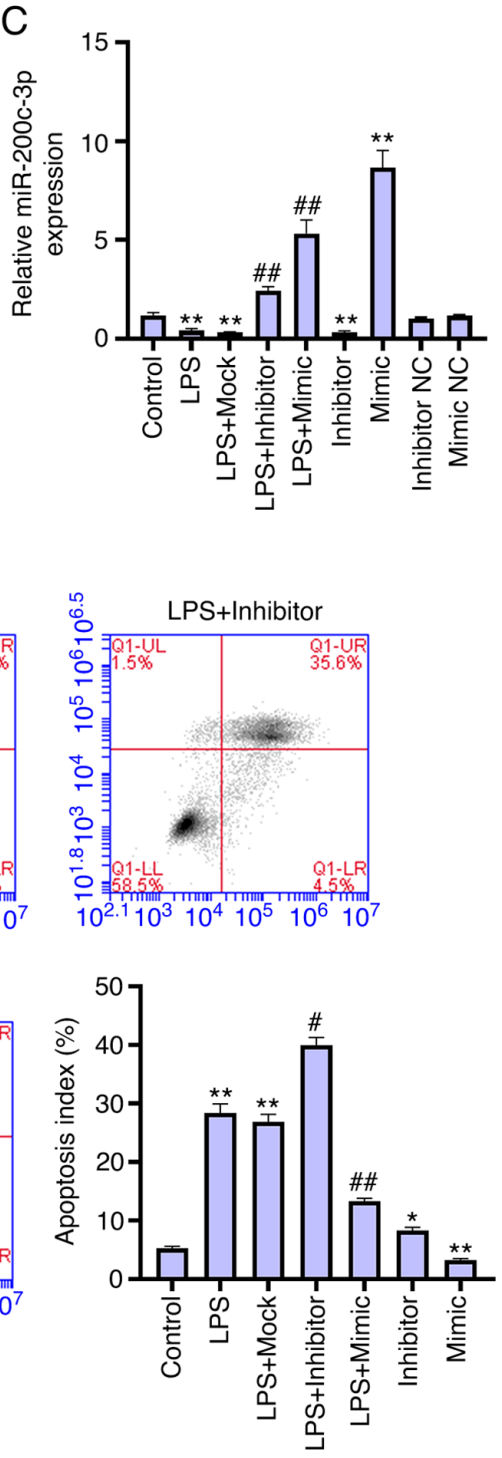

Figure 1. miR-200c-3p inhibits LPS-induced NP cell apoptosis. (A) Expression levels of miR-200c-3p were measured via RT-qPCR in the intervertebral disc tissues of patients with IDD ( $n=22)$ and normal cases $(n=9)$. (B) Expression levels of miR-200c-3p were assessed via RT-qPCR in NP cells treated with LPS $(1 \mu \mathrm{g} / \mathrm{ml})$. ${ }^{*} \mathrm{P}<0.05,{ }^{* *} \mathrm{P}<0.01 \mathrm{vs}$. NC. NP cells were treated with LPS $(1 \mu \mathrm{g} / \mathrm{ml})$ or co-treated with LPS $(1 \mu \mathrm{g} / \mathrm{ml})$ and miR-200c-3p mimic, inhibitor or corresponding control. (C) Expression levels of miR-200c-3p were analyzed via RT-qPCR. (D) Cell apoptosis was tested using flow cytometry analysis. Data are presented as the mean $\pm \mathrm{SD}$. ${ }^{*} \mathrm{P}<0.05,{ }^{* *} \mathrm{P}<0.01$ vs. control; ${ }^{\#} \mathrm{P}<0.05,{ }^{\# \#} \mathrm{P}<0.01$ vs. LPS. LPS, lipopolysaccharide; RT-qPCR, reverse transcription-quantitative PCR; miR, microRNA; NC, negative control; IDD, intervertebral disc degeneration; NP, nucleus pulposus.

CAATACGACCAAATCAGAG-3'; and U6 NCBI RefSeq, NC_015438.3; U6 forward, 5'-GCTTCGGCAGCACATATA CTAA-3' and reverse, 5'-AACGCTTCACGAATTTGCGT-3'.

ELISA. The levels of TNF- $\alpha$ (cat. no. KHC3013), IL-6 (cat. no. KAC1261) and IL-1 $\beta$ (cat. no. KAC1211) were analyzed using ELISA kits (eBioscience; Thermo Fisher Scientific, Inc.) according to the manufacturer's instructions. All standards and samples were tested on a SpectraMax M5 microplate reader device (Molecular Devices, LLC) at an absorbance of $450 \mathrm{~nm}$. A standard curve was prepared, and the concentration of the samples was calculated according to the absorbance value. Data are presented as the mean $\pm \mathrm{SD}$ of three experiments.

Analysis of cell apoptosis. In total, $\sim 2 \times 10^{5} \mathrm{NP}$ cells were plated into 6-well plates. Apoptosis was analyzed using the Annexin V-FITC Apoptosis Detection kit (Cell Signaling
Technology, Inc.) according to the manufacturer's instructions. Briefly, $\sim 2 \times 10^{5} \mathrm{NP}$ cells were collected and washed with binding buffer, followed by flow cytometry analysis on a flow cytometer (BD Accuri ${ }^{\mathrm{TM}}$ C6 Plus; BD Biosciences). The apoptotic rate was calculated as the percentage of early + late apoptotic cells using FlowJo software (v10.7; FlowJo, LLC). Data are presented as the mean $\pm \mathrm{SD}$ of three experiments.

Luciferase reporter gene assay. TargetScan (http://www. targetscan.org/vert_72) was used to verify the potential interaction between RAP2C and miR-200c-3p. Luciferase reporter gene assays were performed using a dual-luciferase reporter assay system (Promega Corporation). The RAP2C wild type or mutant 3'-untranslated region sequences containing the miR-200c-3p binding site were constructed and subcloned into the psiCHECK2 vector (Promega Corporation). Briefly, the miR-200c-3p or control mimic, and the vector containing 
A

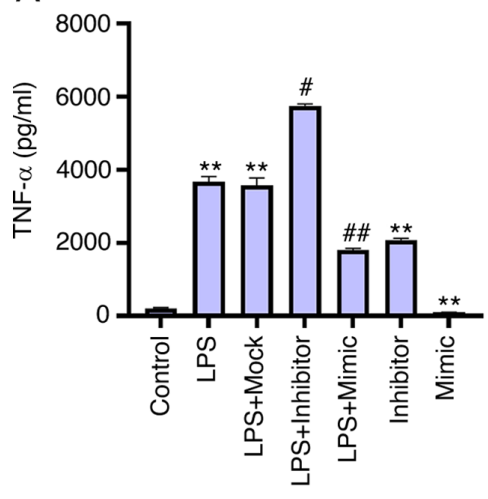

B

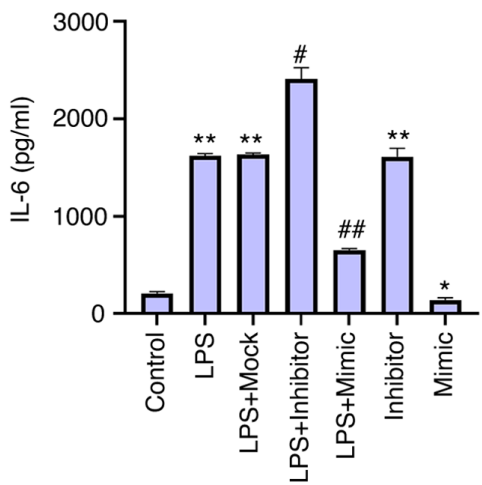

C

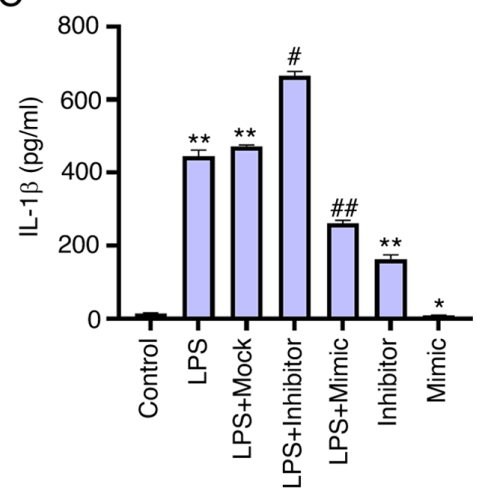

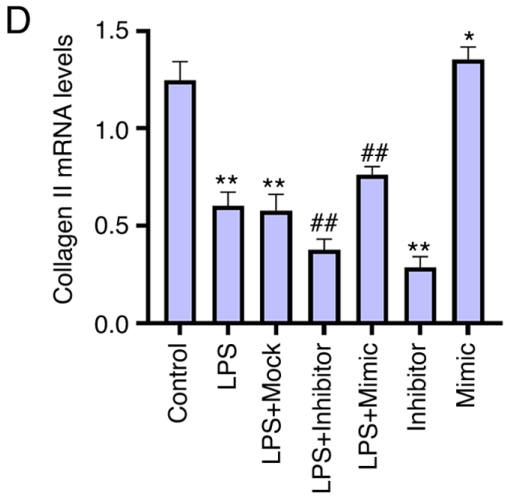

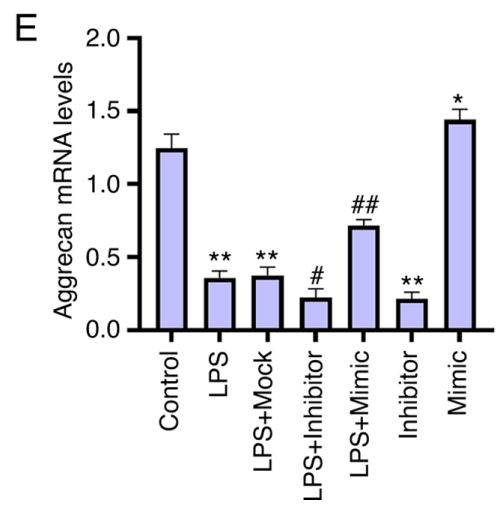

Figure 2. miR-200c-3p attenuates inflammatory cytokine levels and extracellular matrix degradation in LPS-treated NP cells. NP cells were treated with LPS $(1 \mu \mathrm{g} / \mathrm{ml})$ or co-treated with LPS $(1 \mu \mathrm{g} / \mathrm{ml})$ and miR-200c-3p mimic, inhibitor. Levels of (A) TNF- $\alpha$, (B) IL-6 and (C) IL-1 $\beta$ in the culture medium of the cells were analyzed using ELISAs. Expression levels of (D) collagen II and (E) aggrecan were examined via reverse transcription-quantitative PCR. Data are presented as the mean $\pm \mathrm{SD}$. ${ }^{*} \mathrm{P}<0.05,{ }^{* *} \mathrm{P}<0.01$ vs. control; ${ }^{\#} \mathrm{P}<0.05,{ }^{\# \#} \mathrm{P}<0.01$ vs. LPS. LPS. LPS, lipopolysaccharide; miR, microRNA; NP, nucleus pulposus.

RAP2C and RAP2C mutant fragments were transfected into the cells $\left(3 \times 10^{5} /\right.$ well) using Lipofectamine 3000 for $48 \mathrm{~h}$, followed by the analysis of luciferase activities, in which Renilla was used as a normalized control reporter. Data are presented as the mean \pm SD of three experiments.

Western blot analysis. Total proteins were extracted from cells using RIPA buffer (Cell Signaling Technology, Inc.). Protein concentrations were measured using the BCA Protein Quantification kit (Abbkine Scientific Co., Ltd.). An equivalent concentration of protein $(50 \mu \mathrm{g} / \mathrm{lane})$ was separated via SDS-PAGE (12\% polyacrylamide gels) and transferred onto PVDF membranes (MilliporeSigma). The membranes were blocked with $5 \%$ milk at $25^{\circ} \mathrm{C}$ for 60 min and incubated overnight at $4^{\circ} \mathrm{C}$ with the primary antibodies for RAP2C $(1: 3,000$; cat. no. ab97805; Abcam), ERK (1:1,000; cat. no. ab32537; Abcam), phosphorylated (p)-ERK (1:1,000; cat. no. ab194776; Abcam) and $\beta$-actin (1:5,000; cat.no. ab8227; Abcam), in which $\beta$-actin served as the control. Then, the goat anti-rabbit IgG H\&L (HRP) secondary antibodies (1:5,000; cat. no. ab7090; Abcam) were added to membranes for $1 \mathrm{~h}$ at $25^{\circ} \mathrm{C}$, followed by visualization using an Odyssey CLx Infrared Imaging system (LI-COR Biosciences). Image Studio ${ }^{\text {TM }}$ Lite (v4.0; LI-COR Biosciences) was used for analysis. Data are presented as the mean \pm SD of three experiments.

Statistical analysis. Data are presented as the mean \pm SD of three experiments and statistical analysis was performed using
GraphPad Prism 7 (GraphPad Software, Inc.). An unpaired Student's t-test was used to compare two groups. One-way ANOVA was used for multiple group comparisons and Tukey's test was used for post hoc analysis. $\mathrm{P}<0.05$ was considered to indicate a statistically significant difference.

\section{Results}

miR-200c-3p is downregulated in IDD and inhibits LPS-induced NP cell apoptosis. To assess the potential association between miR-200c-3p and IDD, the expression level of miR-200c-3p was analyzed in patients with IDD and normal controls. The expression level of miR-200c-3p was decreased in the intervertebral disc tissues of patients with IDD $(n=22)$ compared with that in normal subjects $(n=9)$ (Fig. 1A), suggesting a potential association between miR-200c-3p and IDD development. Next, the expression of miR-200c-3p was examined in LPS-treated NP cells. LPS treatment reduced the expression of miR-200c-3p in NP cells in a time-dependent manner (Fig. 1B), indicating that miR-200c-3p may be involved in IDD progression. NP cell incubation with LPS for $24 \mathrm{~h}$ was selected for the next experiment.

To evaluate the role of miR-200c-3p in the development of IDD in vitro, LPS-treated NP cells were transfected with miR-200c-3p mimic or miR-200c-3p inhibitor and the efficiency was validated (Fig. 1C). As expected, LPS treatment increased the apoptosis of NP cells, in which the miR-200c-3p inhibitor enhanced, while the miR-200c-3p mimic suppressed 

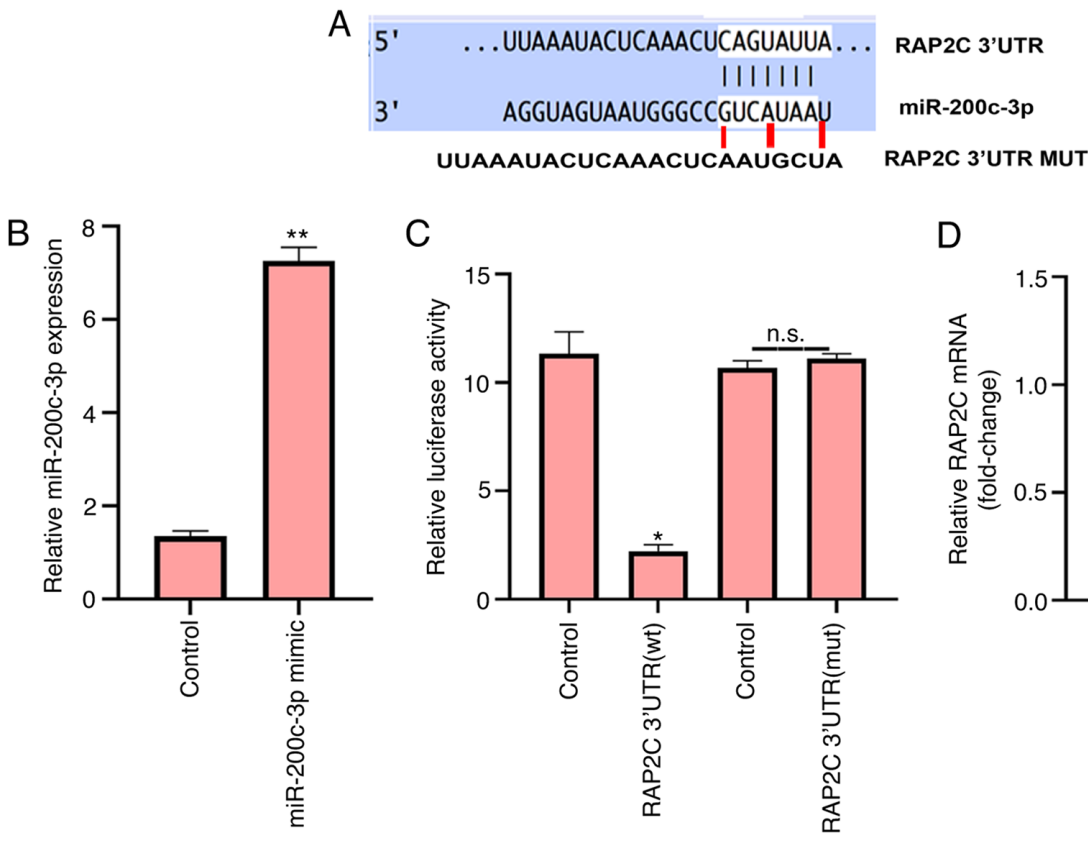

C
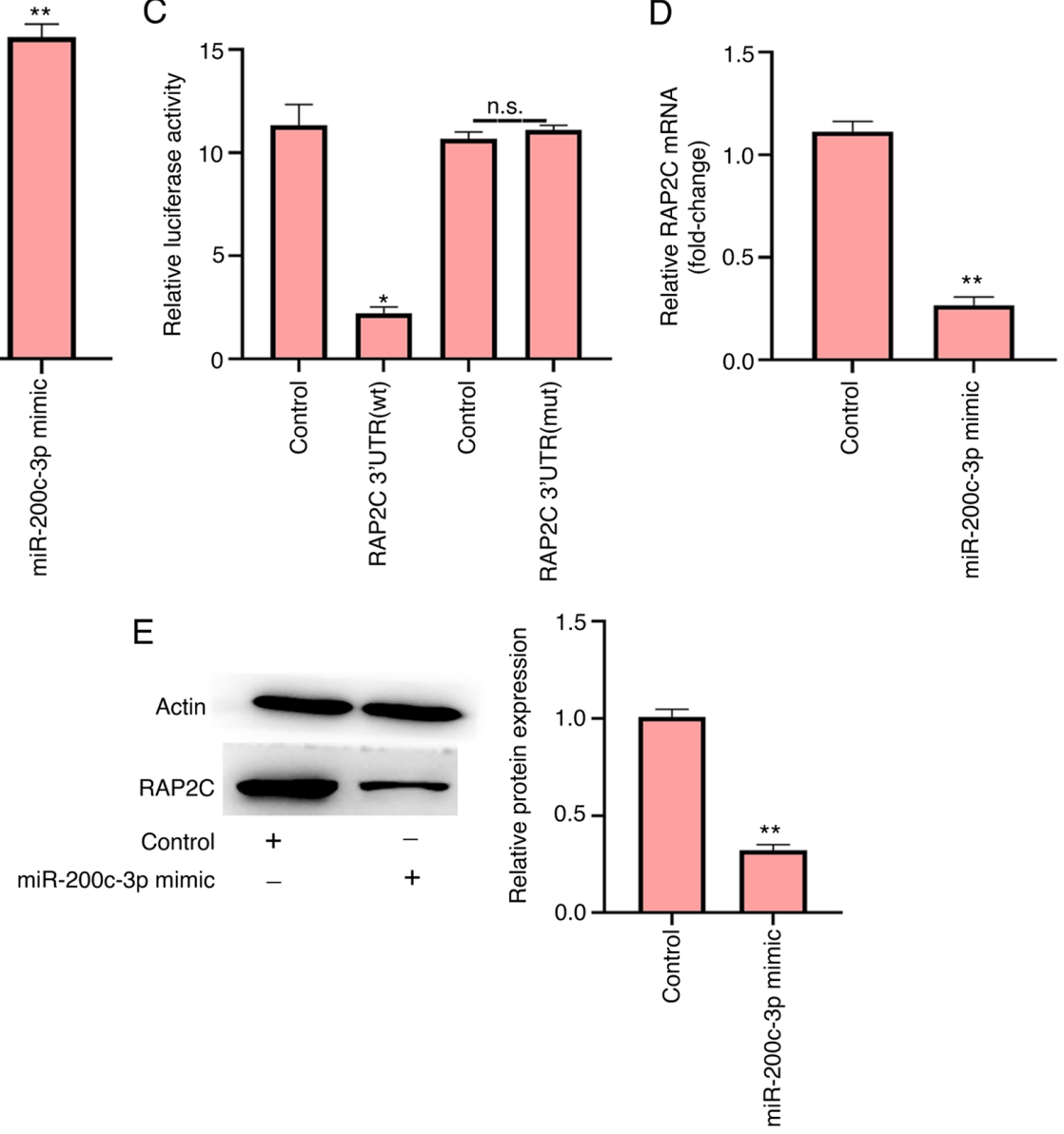

Figure 3. miR-200c-3p targets RAP2C in nucleus pulposus cells. (A) Interaction of miR-200c-3p and RAP2C 3'UTR was identified via bioinformatics analysis using TargetScan. (B) NP cells were transfected with control mimic or miR-200c-3p mimic. (C) Luciferase activities of RAP2C WT and RAP2C with the miR-200c-3p-binding site mutant (RAP2C MUT) were determined using luciferase reporter gene assays. (D) mRNA expression level of RAP2C was assessed using reverse transcription-quantitative PCR in the cells. (E) Protein expression level of RAP2C was measured via western blotting. Data are presented as the mean $\pm \mathrm{SD} .{ }^{*} \mathrm{P}<0.05,{ }^{* *} \mathrm{P}<0.01$ vs. control. ns, no significance; miR, microRNA; WT, wild-type; MUT, mutant; RAP2C, Ras-related protein $2 \mathrm{C}$; UTR, untranslated region.

this effect (Fig. 1D), suggesting that miR-200c-3p inhibits LPS-induced NP cell apoptosis.

miR-200c-3p attenuates inflammatory cytokine levels and ECM degradation in LPS-treated NP cells. Since inflammatory factors have been shown to be involved in the pathological processes of IDD (21). The effect of miR-200c-3p on the levels of inflammatory factors was determined in LPS-treated NP cells. The levels of inflammatory cytokines in the culture medium of NP cells, including TNF- $\alpha$, IL- 6 and IL- $1 \beta$, were increased by LPS stimulation, in which the miR-200c-3p inhibitor enhanced, but the miR-200c-3p mimic reduced this phenotype in the cells (Fig. 2A-C). This suggests that miR-200c-3p inhibits inflammatory cytokine levels in LPS-treated NP cells. Moreover, the expression levels of collagen II and aggrecan were significantly decreased by the miR-200c-3p inhibitor but were upregulated by the miR-200c-3p mimic in the LPS-treated NP cells (Fig. 2D and E), indicating that
miR-200c-3p attenuates ECM degradation in LPS-treated NP cells.

miR-200c-3p targets RAP2C in NP cells. miR-200c-3p has a recognized target site in RAP2C 3'UTR, as determined using TargetScan (Fig. 3A). To examine the effect of miR-200c-3p on RAP2C, the NP cells were transfected with miR-200c-3p mimic (Fig. 3B). Notably, the miR-200c-3p mimic inhibited luciferase activity of wild-type RAP2C but failed to affect the RAP2C with the miR-200c-3p-binding site mutant in the NP cells (Fig. 3C). Furthermore, the mRNA and protein expression levels of RAP2C were significantly downregulated by miR-200c-3p mimic transfection in NP cells (Fig. 3D and E), suggesting that miR-200c-3p can target RAP2C in NP cells.

$R A P 2 C$ promotes apoptosis, inflammatory cytokine levels and ECM degradation in LPS-treated NP cells. LPS-treated NP 
A

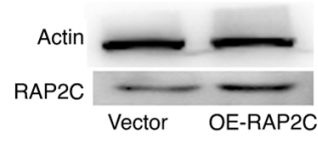

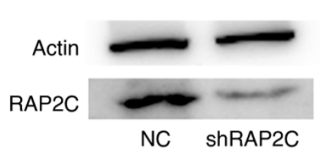

NC ShRAP2C
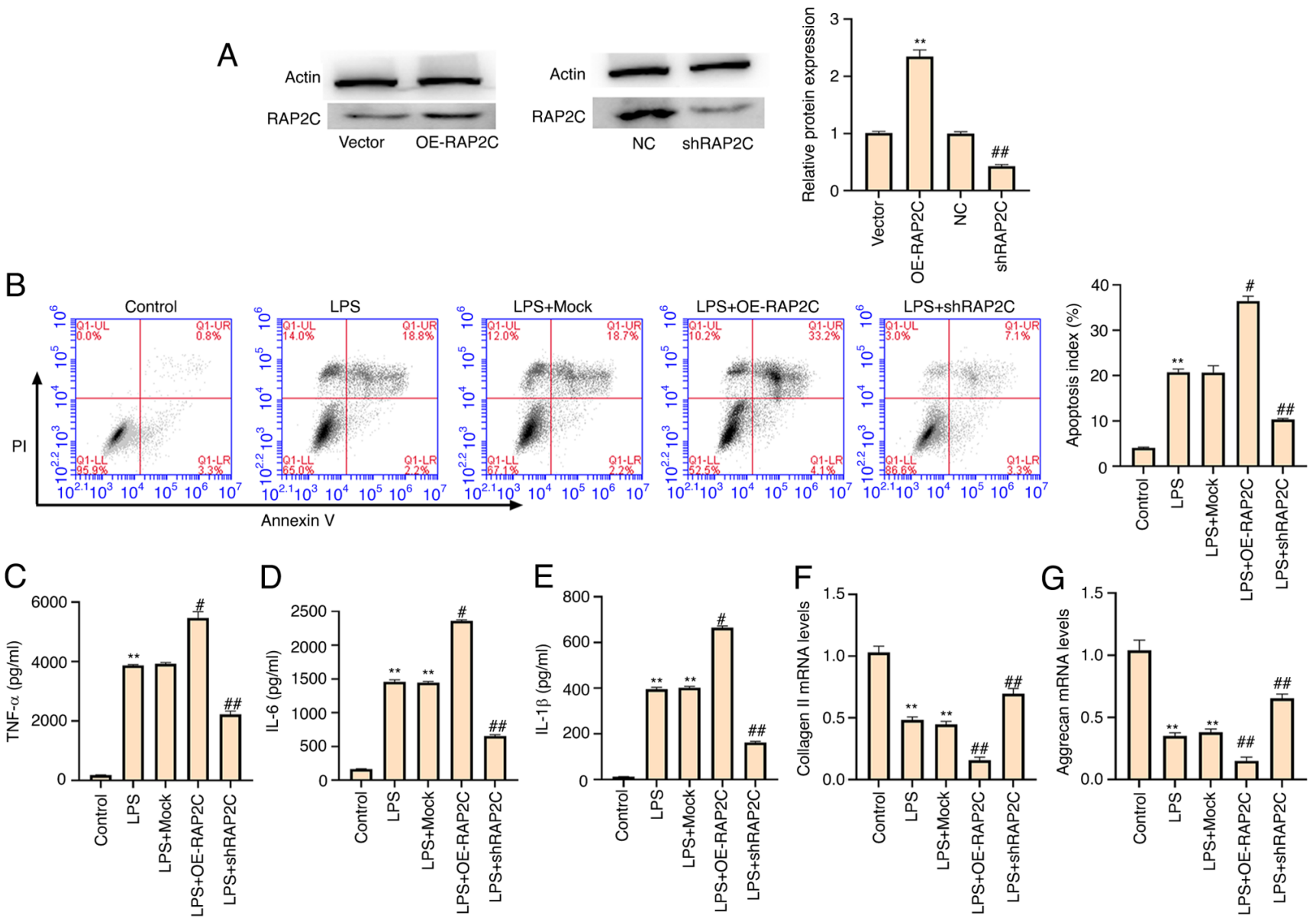

Figure 4. RAP2C promotes apoptosis, inflammatory cytokine levels and ECM degradation in LPS-treated NP cells. LPS (1 $\mu$ g/ml)-treated NP cells were infected with lentiviral plasmids carrying RAP2C shRNA or the corresponding control shRNA, or transfected with the control vector or the vector carrying the complete RAP2C coding sequence for overexpression. (A) Expression levels of RAP2C were measured via western blotting. ${ }^{* *} \mathrm{P}<0.01$ vs. vector; ${ }^{\# \#} \mathrm{P}<0.01$ vs. NC. (B) Cell apoptosis was measured using flow cytometry analysis. Levels of (C) TNF- $\alpha$, (D) IL-6 and (E) IL-1 $\beta$ in the culture medium of the cells were analyzed using ELISAs. Expression levels of (F) collagen II and (G) aggrecan were examined via reverse transcription-quantitative PCR. Data are presented as the mean $\pm \mathrm{SD} .{ }^{* *} \mathrm{P}<0.01$ vs. control; ${ }^{\#} \mathrm{P}<0.05,{ }^{\# \#} \mathrm{P}<0.01$ vs. LPS. LPS, lipopolysaccharide; shRNA/sh, short hairpin RNA; NC, negative control; RAP2C, Ras-related protein 2C; NP, nucleus pulposus; OE, overexpression vector.

cells were infected with lentiviral plasmids carrying RAP2C shRNA or corresponding control shRNA or transfected with the vector carrying the complete RAP2C coding sequence for overexpression or the control vector. The efficiency of RAP2C shRNA and RAP2C overexpression was validated in the cells (Fig. 4A). It was demonstrated that the LPS-induced apoptosis of NP cells was significantly enhanced by RAP2C overexpression and reduced by RAP2C knockdown (Fig. 4B), suggesting that RAP2C promotes NP cell apoptosis. Moreover, the upregulation of TNF- $\alpha$, IL- 6 and IL-1 $\beta$ by LPS treatment was increased by the overexpression of RAP2C but was suppressed by RAP2C knockdown in the NP cells (Fig. 4C-E), indicating that RAP2C activates the inflammatory cytokine levels. Furthermore, RAP2C overexpression inhibited and RAP2C knockdown increased the expression levels of collagen II and aggrecan in NP cells (Fig. 4F and G), suggesting that RAP2C contributes to ECM degradation in LPS-treated NP cells.

$R A P 2 C$ enhances apoptosis, inflammatory cytokine levels and ECM degradation by activating ERK signaling. It has been reported that the activation of ERK signaling contributes to the development of IDD $(14,22)$. The phosphorylation of ERK is required for activation of ERK signaling $(23,24)$. The expression level of RAP2C was significantly upregulated by the overexpression of RAP2C, whereas the ERK inhibitor SCH772984 partially reduced this upregulation in NP cells. Moreover, the knockdown of RAP2C significantly decreased RAP2C expression (Fig. 5A). The knockdown of RAP2C increased the ratio of p-/total ERK protein expression (Fig. 5A). Moreover, RAP2C induced LPS-treated NP cell apoptosis, and $\mathrm{SCH} 772984$ partially restored this phenotype (Fig. 5B). In addition, SCH772984 partially blocked the elevated levels of TNF- $\alpha$, IL- 6 and IL- $1 \beta$ caused by the overexpression of RAP2C in LPS-treated NP cells (Fig. 5C-E). In addition, RAP2C overexpression-inhibited expression of collagen II and aggrecan was rescued by SCH772984 treatment in LPS-treated NP cells (Fig. 5F and G). Taken together, these data suggest that RAP2C enhances apoptosis, inflammatory cytokine levels and ECM degradation by activating ERK signaling.

miR-200c-3p suppresses IDD by targeting RAP $2 C / E R K$ signaling. Knockdown of miR-200c-3p with a miR-200c-3p inhibitor promoted the expression of RAP2C. However, 

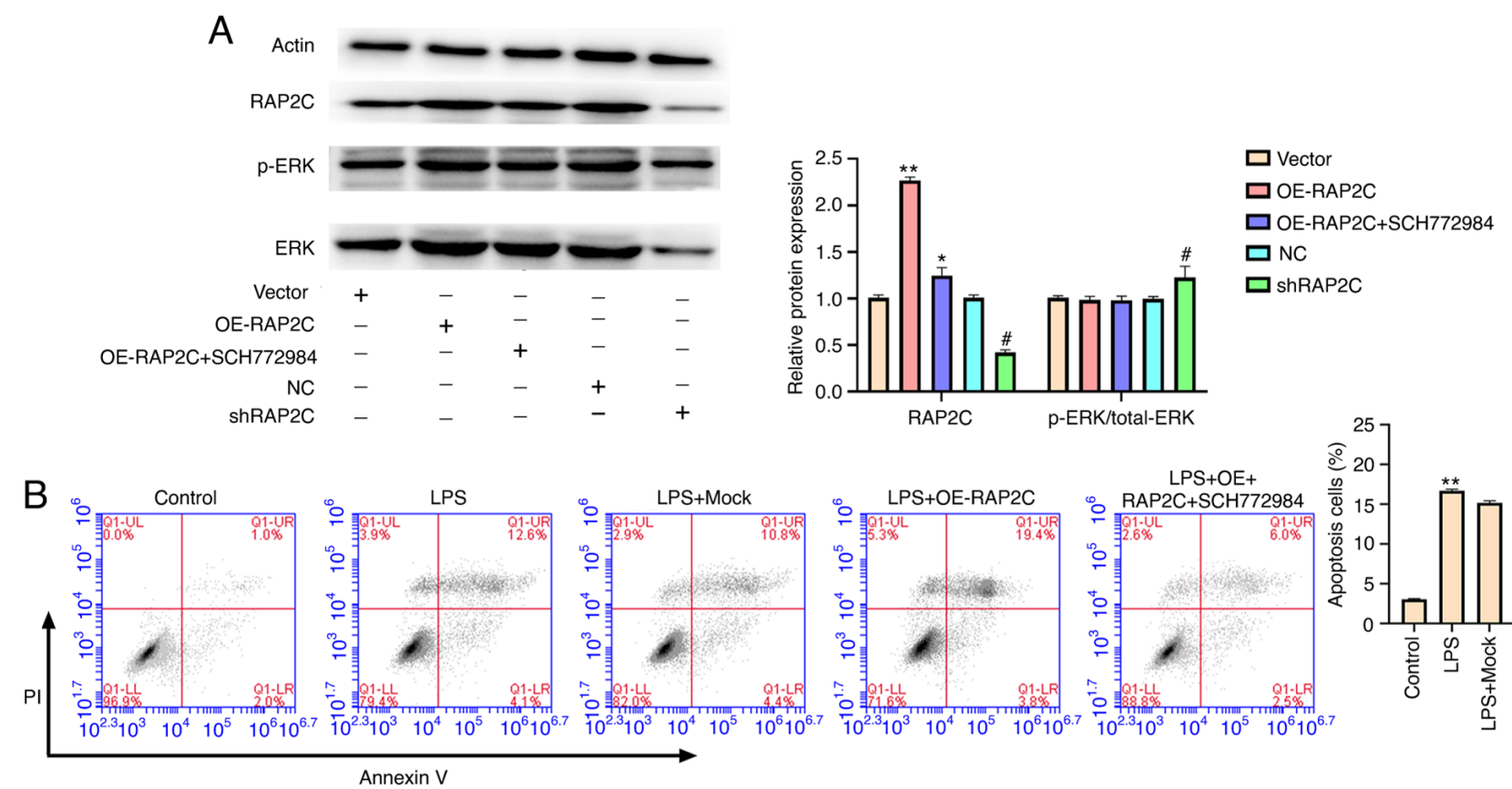

-ERK/total-ERK
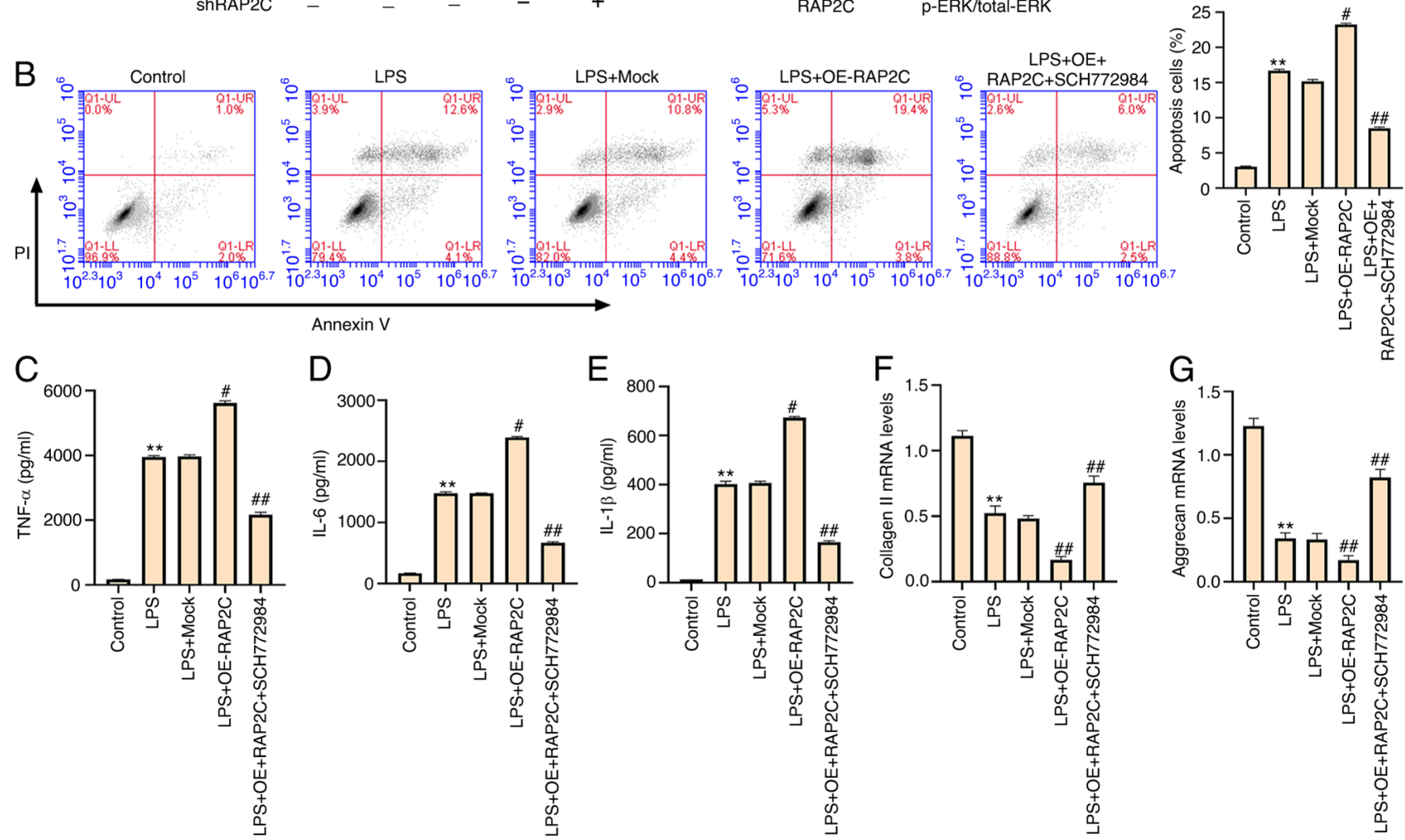

Figure 5. RAP2C enhances apoptosis, inflammatory cytokine levels and extracellular matrix degradation by activating ERK signaling. (A) NP cells were infected with the lentiviral plasmids carrying RAP2C shRNA or the corresponding control shRNA, transfected with the control vector or the vector carrying the complete RAP2C coding sequence for overexpression or co-treated with the RAP2C overexpression vector and the ERK inhibitor SCH772984 (10 $\mu$ mol/l). The expression levels of RAP2C, ERK and $\beta$-actin, and the phosphorylation of ERK (p-ERK) were examined via western blotting. ${ }^{*} \mathrm{P}<0.05$, ${ }^{* * *} \mathrm{P}<0.01$ vs. vector; ${ }^{\text {"P }}<0.05$ vs. NC. LPS $(1 \mu \mathrm{g} / \mathrm{ml})$-treated NP cells were transfected with the control vector, or the vector carrying the complete RAP2C coding sequence for overexpression or co-treated with RAP2C overexpression vector and the ERK inhibitor SCH772984 (10 $\mu \mathrm{mol} / \mathrm{l})$. (B) Cell apoptosis was examined via flow cytometry analysis. Levels of (C) TNF- $\alpha$, (D) IL-6 and (E) IL-1 $\beta$ in the culture medium of the cells were tested using ELISAs. Expression levels of (F) collagen II and $(\mathrm{G})$ aggrecan were determined using reverse transcription-quantitative PCR. Data are presented as the mean $\pm \mathrm{SD}$. ${ }^{*} \mathrm{P}<0.05$, ${ }^{* *} \mathrm{P}<0.01 \mathrm{vs}$. control; ${ }^{*} \mathrm{P}<0.05$, ${ }^{\# \#} \mathrm{P}<0.01$ vs. LPS. LPS, lipopolysaccharide; shRNA/sh, short hairpin RNA; NC, negative control; RAP2C, Ras-related protein 2C; NP, nucleus pulposus; OE, overexpression vector; p-, phosphorylated.

SCH772984 treatment blocked miR-200c-3p inhibitor-induced RAP2C expression in NP cells. The miR-200c-3p mimic inhibited the expression of RAP2C (Fig. 6A). The miR-200c-3p mimic also increased the ratio of $\mathrm{p}$-/total ERK protein expression (Fig. 6A). Moreover, the miR-200c-3p inhibitor-induced apoptosis of LPS-treated NP cells was partially inhibited by RAP2C shRNA or SCH772984 (Fig. 6B). In addition, the levels of TNF- $\alpha$, IL- 6 and IL- $1 \beta$ enhanced by the miR-200c-3p inhibitor were partially decreased by RAP2C knockdown or SCH772984 treatment in NP cells (Fig. 6C-E). RAP2C knockdown and SCH772984 treatment also partially restored the expression levels of collagen II and aggrecan reduced by miR-200c-3p inhibitor (Fig. 6F and G). Collectively, these results suggest that miR-200c-3p suppresses IDD by targeting RAP2C/ERK signaling.

\section{Discussion}

IDD is a complex disorder characterized by genotypic and phenotypic alterations, such as ECM degradation, apoptosis and inflammation (25). miRNAs are essential regulators of multiple physiological and pathological processes, and are involved in the modulation of IDD progression. It has been reported that TLR4/NF- $\kappa B$ signaling increases the expression of miR-625-5p, thereby contributing to the pathological process of IDD by modulating collagen type I $\alpha 1$ (26). 
A

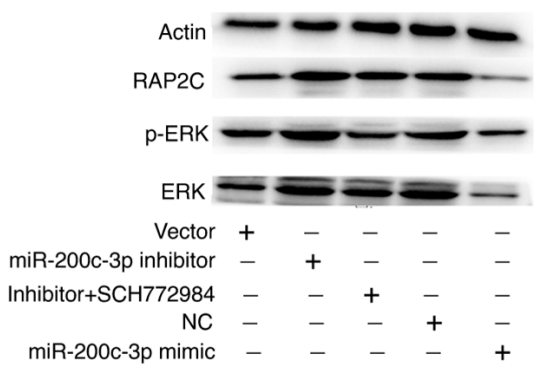

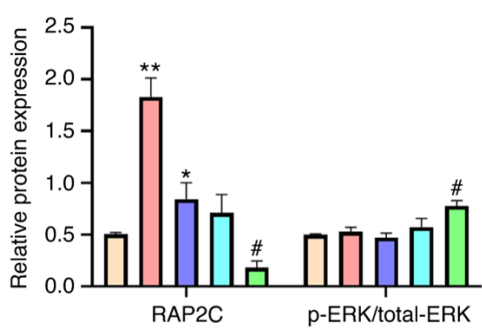

$\square$ Vector

$\square$ miR-200c-3p inhibitor

$\square$ Inhibitor+SCH772984

$\square$ NC

$\square$ miR-200c-3p mimic

B
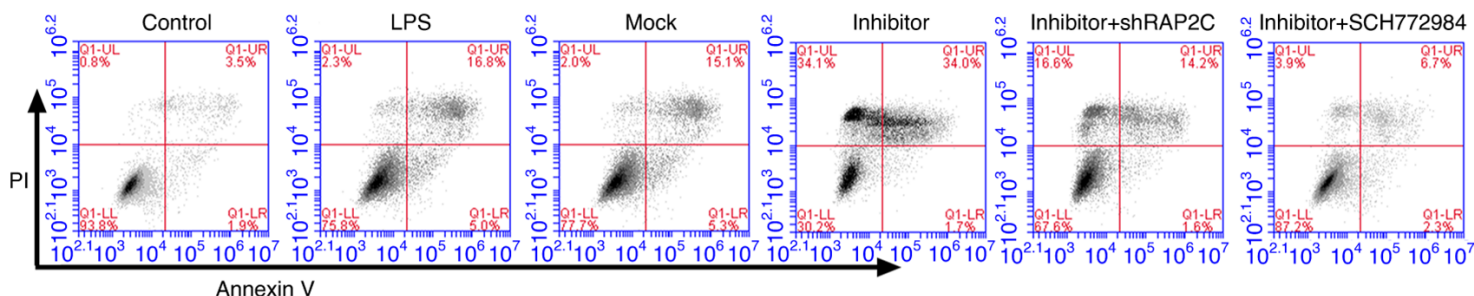

Annexin V

C

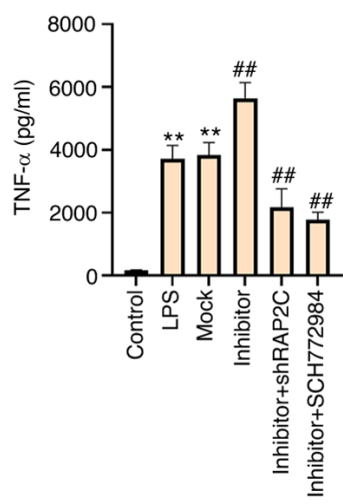

D

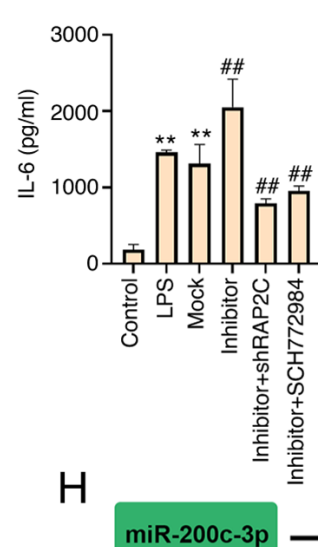

E

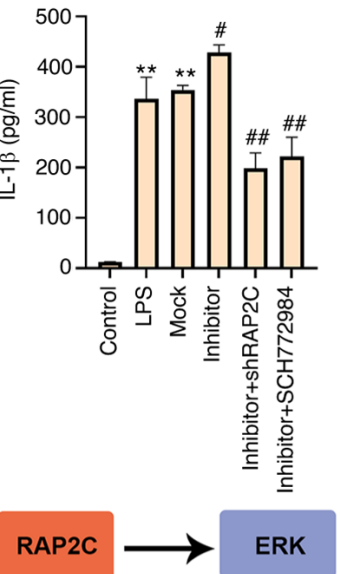

F

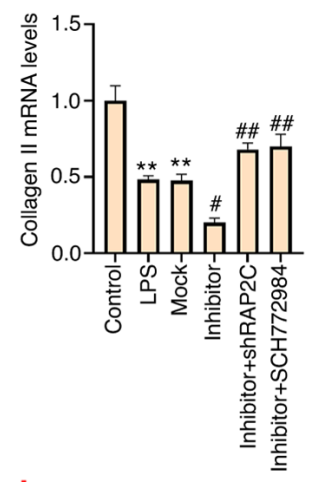

G

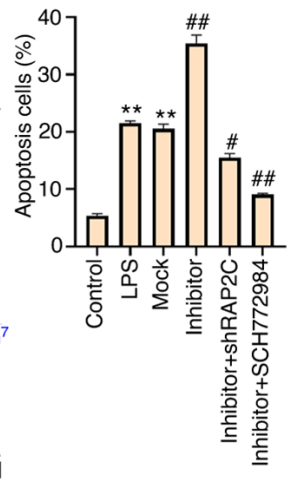

Figure 6. miR-200c-3p suppresses IDD by targeting RAP2C/ERK signaling. (A) NP cells were transfected with miR-200c-3p mimic, inhibitor or corresponding control, or co-treated with miR-200c-3p inhibitor and the ERK inhibitor SCH772984 (10 $\mu$ mol/1). The expression levels of RAP2C, ERK and $\beta$-actin, and the phosphorylation of ERK (p-ERK) were examined via western blotting. LPS ( $\mu \mathrm{g} / \mathrm{ml})$-treated NP cells were transfected with miRNA control inhibitor or miR-200c-3p inhibitor, or co-transfected with miR-200c-3p inhibitor and the lentiviral plasmids carrying RAP2C shRNA, or co-transfected with miR-200c-3p inhibitor and the ERK inhibitor SCH772984 (10 $\mu \mathrm{mol} / 1) .{ }^{*} \mathrm{P}<0.05,{ }^{* *} \mathrm{P}<0.01$ vs. vector; ${ }^{*} \mathrm{P}<0.05$ vs. NC. (B) Cell apoptosis was analyzed using flow cytometry. The levels of (C) TNF- $\alpha$, (D) IL-6 and (E) IL-1 $\beta$ in the culture medium of the cells were assessed using ELISA. The expression levels of (F) collagen II and $(\mathrm{G})$ aggrecan were examined using reverse transcription-quantitative PCR. (H) The regulatory pathway of miR-200c-3p in LPS-treated NP cells. Data are presented as the mean $\pm \mathrm{SD} .{ }^{* *} \mathrm{P}<0.01$ vs. control; ${ }^{\#} \mathrm{P}<0.05,{ }^{\#} \mathrm{P}<0.01 \mathrm{vs}$. LPS. IDD, intervertebral disc degeneration; LPS, lipopolysaccharide; shRNA/sh, short hairpin RNA; NC, negative control; RAP2C, Ras-related protein 2C; NP, nucleus pulposus; p-, phosphorylated; miRNA/miR, microRNA.

miR-665 contributes to the proliferation and matrix degradation of NP cells by regulating the expression of GDF5 in IDD (27). Moreover, miR-24-3p upregulation is able to increase IDD by targeting insulin-like growth factor-binding protein 5 and ERK signaling (28), while miR-640 induces IDD by modulating Wnt and NF- $\kappa \mathrm{B}$ signaling (29). Contrary to the aforementioned results, miR-200c-3p expression was significantly decreased in the intervertebral disc tissues of patients with IDD and it inhibited apoptosis, inflammatory cytokine levels and ECM degradation in IDD by targeting RAP2C/ERK signaling.

RAP2C is an evolutionarily conserved Ras-like GTPase that serves as a molecular switch that pairs receptor signaling to remodel the actin cytoskeleton, cell adhesion and cell polarity $(30,31)$. Previous studies have identified a correlation between miRNAs and RAP2C in multiple pathological processes $(10,32,33)$. Spinal cord ischemia/reperfusion injury promotes neurocyte apoptosis by reducing RAP2C via sponging miR-204 (34). miR-188-5p increases apoptosis and represses proliferation in breast cancer cells by regulating RAP2C/MAPK signaling (35). In addition, it has been shown that RAP2C can activate ERK signaling (12). Similarly, in the present study, RAP2C was targeted by miR-200c-3p in NP cells, whereby RAP2C promoted apoptosis, inflammatory cytokine levels and ECM degradation in LPS-treated NP cells by activating ERK signaling.

ECM degradation plays a critical role in the development of IDD, in which collagen II and aggrecan serve as markers of ECM degradation (36). In the present study, the expression levels of collagen II and aggrecan were decreased 
by miR-200c-3p inhibitors but upregulated by miR-200c-3p mimics in LPS-treated NP cells. This finding was in agreement with a previous study showing that long non-coding RNA nuclear paraspeckle assembly transcript 1 downregulated aggrecan, collagen II, thrombospondin-type motifs 5 and MMP13 by modifying ERK/MAPK signaling in NP cells (37). Consistently, TGF- $\beta 1$ inhibits the expression of C-C motif chemokine ligand $3 / 4$ via ERK signaling and restricts IDD and inflammation-induced pain (38). Moreover, autophagy alleviates compression-related NP cell apoptosis in IDD via $\mathrm{MEK} / \mathrm{ERK} /$ nuclear respiratory factor 1/autophagy related 7 signaling (39). The present study demonstrated that ERK signaling was involved in the miR-200c-3p/RAP2C-mediated apoptosis, inflammatory cytokine levels and ECM degradation in NP cells.

The current study has some limitations. The expression levels of aggrecan and collagen II were determined only via RT-qPCR, while levels of inflammatory factors were assessed using ELISA alone. In future studies, the results of the present study should be verified using in vivo models and the regulation of IDD by miR-200c-3p requires further investigation.

In conclusion, the present study demonstrated that miR-200c-3p inhibited NP cell apoptosis, inflammatory cytokine levels and ECM degradation in IDD by targeting RAP2C/ERK signaling. Moreover, miR-200c-3p and RAP2C may serve as potential targets for IDD therapy.

\section{Acknowledgements}

Not applicable.

\section{Funding}

This study was supported in part by the Qingdao Medical Research Guidance Program in 2018: Three-dimensional Decompression Comprehensive Therapy for the Treatment of Lumbar Disc Herniation and Degeneration (grant no. 2018-WJZD113).

\section{Availability of data and materials}

The datasets used and/or analyzed during the current study are available from the corresponding author on reasonable request.

\section{Authors' contributions}

JC and KX designed the study. MJ performed the experiments. $\mathrm{HR}$ analyzed data. JC and KX confirm the authenticity of all the raw data. KX wrote the paper. All authors read and approved the final manuscript.

\section{Ethics approval and consent to participate}

The protocol of this research has been approved by the Ethics Committee of Qingdao No.6 People's Hospital. All patients have signed written informed consent.

\section{Patient consent for publication}

Not applicable.

\section{Competing interests}

The authors declare that they have competing interests.

\section{References}

1. Wang SZ, Chang Q, Lu J and Wang C: Growth factors and platelet-rich plasma: Promising biological strategies for early intervertebral disc degeneration. Int Orthop 39: 927-934, 2015.

2. Tisherman R, Coelho P, Phillibert D, Wang D, Dong Q, Vo N, Kang $\mathrm{J}$ and Sowa G: NF- $\mathrm{BB}$ signaling pathway in controlling intervertebral disk cell response to inflammatory and mechanical stressors. Phys Ther 96: 704-711, 2016.

3. Zhang Y, Yang J, Zhou X, Wang N, Li Z, Zhou Y, Feng J, Shen D and Zhao W: Knockdown of miR-222 inhibits inflammation and the apoptosis of LPS-stimulated human intervertebral disc nucleus pulposus cells. Int J Mol Med 44: 1357-1365, 2019.

4. Zhou Q, Huang SX, Zhang F, Li SJ, Liu C, Xi YY, Wang L, Wang X, He QQ, Sun CC and Li DJ: MicroRNAs: A novel potential biomarker for diagnosis and therapy in patients with non-small cell lung cancer. Cell Prolif 50: e12394, 2017.

5. Zhang Q, Weng Y, Jiang Y, Zhao S, Zhou D and Xu N: Overexpression of miR-140-5p inhibits lipopolysaccharide-induced human intervertebral disc inflammation and degeneration by downregulating toll-like receptor 4 . Oncol Rep 40: 793-802, 2018.

6. Liu W, Xia P, Feng J, Kang L, Huang M, Wang K, Song Y, Li S, Wu X, Yang S and Yang C: MicroRNA-132 upregulation promotes matrix degradation in intervertebral disc degeneration. Exp Cell Res 359: 39-49, 2017.

7. Yu G, Jiao Y, Huang JJ, Fan MD, Hao YC, Han JZ and Qu L: Acidic preconditioning reduces lipopolysaccharide-induced acute lung injury by upregulating the expression of angiotensin-converting enzyme 2. Exp Ther Med 21: 441, 2021.

8. LaiZ and Cao Y: Plasma miR-200c-3p, miR-100-5p, and miR-1826 serve as potential diagnostic biomarkers for knee osteoarthritis: Randomized controlled trials. Medicine (Baltimore) 98: e18110, 2019.

9. Chen P, Jiang P, Chen J, Yang Y and Guo X: XIST promotes apoptosis and the inflammatory response in CSE-stimulated cells via the miR-200c-3p/EGR3 axis. BMC Pulm Med 21: 021-01582, 2021

10. Wang Z, Huang C, Zhang A, Lu C and Liu L: Overexpression of circRNA_100290 promotes the progression of laryngeal squamous cell carcinoma through the miR-136-5p/RAP2C axis. Biomed Pharmacother 125: 109874, 2020.

11. Wu J, Du W, Wang X, Wei L, Pan Y, Wu X, Zhang J and Pei D: Ras-related protein Rap2c promotes the migration and invasion of human osteosarcoma cells. Oncol Lett 15: 5352-5358, 2018.

12. Wang Z, Huang C, Zhang A, Lu C and Liu L: Overexpression of circRNA_100290 promotes the progression of laryngeal squamous cell carcinoma through the miR-136-5p/RAP2C axis. Biomed Pharmacother 125: 10987, 2020.

13. Wang X, Wang C, Xi L and Yu Z: Rap2c as a novel biomarker for predicting poor prognosis in glioma. Onco Targets Ther 13: 3073-3083, 2020.

14. Mi D, Cai C, Zhou B, Liu X, Ma P, Shen S, Lu W and Huang W: Long noncoding RNA FAF1 promotes intervertebral disc degeneration by targeting the Erk signaling pathway. Mol Med Rep 17: 3158-3163, 2018.

15. Li L, Wei K, Ding Y, Ahati P, Xu H, Fang H and Wang H: M2a macrophage-secreted $\mathrm{CHI} 3 \mathrm{~L} 1$ promotes extracellular matrix metabolic imbalances via activation of IL-13R $\alpha 2 /$ MAPK pathway in rat intervertebral disc degeneration. Front Immunol 12: 666361, 2021.

16. Ni L, Zheng Y, Gong T, Xiu C, Li K, Saijilafu, Li B, Yang H and Chen J: Proinflammatory macrophages promote degenerative phenotypes in rat nucleus pulpous cells partly through ERK and JNK signaling. J Cell Physiol 234: 5362-5371, 2019.

17. Pfirrmann CW, Metzdorf A, Zanetti M, Hodler J and Boos N: Magnetic resonance classification of lumbar intervertebral disc degeneration. Spine (Phila Pa 1976) 26: 1873-1878, 1976.

18. Ji Y, Hong W, Liu M, Liang Y, Deng Y and Ma L: Intervertebral disc degeneration associated with vertebral marrow fat, assessed using quantitative magnetic resonance imaging. Skeletal Radiol 49: 1753-1763, 2020. 
19. Livak KJ and Schmittgen TD: Analysis of relative gene expression data using real-time quantitative PCR and the 2(-Delta Delta C(T)) method. Methods 25: 402-408, 2001

20. Yang Y, Zhong Z, Zhao Y, Ren K and Li N: LincRNA-SLC20A1 (SLC20A1) promotes extracellular matrix degradation in nucleus pulposus cells in human intervertebral disc degeneration by targeting the miR-31-5p/MMP3 axis. Int J Clin Exp Pathol 12: 3632-3643, 2019

21. Navone SE, Marfia G, Giannoni A, Beretta M, Guarnaccia L, Gualtierotti R, Nicoli D, Rampini P and Campanella R: Inflammatory mediators and signalling pathways controlling intervertebral disc degeneration. Histol Histopathol 32: 523-542, 2017.

22. Han YC, Ma B, Guo S, Yang M, Li LJ, Wang SJ and Tan J: Leptin regulates disc cartilage endplate degeneration and ossification through activation of the MAPK-ERK signalling pathway in vivo and in vitro. J Cell Mol Med 22: 2098-2109, 2018.

23. Roskoski R Jr: ERK1/2 MAP kinases: Structure, function, and regulation. Pharmacol Res 66: 105-143, 2012.

24. Roskoski R Jr: Targeting ERK1/2 protein-serine/threonine kinases in human cancers. Pharmacol Res 142: 151-168, 2019.

25. Sampara P, Banala RR, Vemuri SK, Av GR and Gpv S Understanding the molecular biology of intervertebral disc degeneration and potential gene therapy strategies for regeneration: A review. Gene Ther 25: 67-82, 2018.

26. Shen L, Xiao Y, Wu Q, Liu L, Zhang C and Pan X: TLR4/NF-кB axis signaling pathway-dependent up-regulation of miR-625-5p contributes to human intervertebral disc degeneration by targeting COL1A1. Am J Transl Res 11: 1374-1388, 2019.

27. Tan H, Zhao L, Song R, Liu Y and Wang L: microRNA-665 promotes the proliferation and matrix degradation of nucleus pulposus through targeting GDF5 in intervertebral disc degeneration. J Cell Biochem 119: 7218-7225, 2018.

28. Chen Z, Liu M, Zhang W, Deng M, Zhou Y and Li Y: miR-24-3p induces human intervertebral disc degeneration by targeting insulin-like growth factor binding protein 5 and the ERK signaling pathway. Life Sci 243: 117288, 2020.

29. Dong W, Liu J, Lv Y, Wang F, Liu T, Sun S, Liao B, Shu Z and Qian J: miR-640 aggravates intervertebral disc degeneration via NF- $\kappa \mathrm{B}$ and WNT signalling pathway. Cell Prolif 52: e12664, 2019.

30. Lagarrigue F, Kim C and Ginsberg MH: The Rap1-RIAM-talin axis of integrin activation and blood cell function. Blood 128: 479-487, 2016
31. Paganini S, Guidetti GF, Catricalà S, Trionfini P, Panelli S, Balduini $\mathrm{C}$ and Torti $\mathrm{M}$ : Identification and biochemical characterization of Rap2C, a new member of the Rap family of small GTP-binding proteins. Biochimie 88: 285-295, 2006.

32. Liao X, Zhan W, Tian B, Luo Y, Gu F and Li R: Circular RNA ZNF609 promoted hepatocellular carcinoma progression by upregulating PAP2C expression via sponging miR-342-3p. Onco Targets Ther 13: 7773-7783, 2020.

33. Shen Z, Zhou R, Liu C, Wang Y, Zhan W, Shao Z, Liu J, Zhang F, $\mathrm{Xu}$ L, Zhou X, et al: MicroRNA-105 is involved in TNF- $\alpha$-related tumor microenvironment enhanced colorectal cancer progression. Cell Death Dis 8: 3213, 2017.

34. Qiao Y, Peng C, Li J, Wu D and Wang X: Spinal cord ischemia-reperfusion causes damage of neurocyte by inhibiting RAP2C. Neurol Res 39: 877-884, 2017.

35. Zhu X, Qiu J, Zhang T, Yang Y, Guo S, Li T, Jiang K, Zahoor A, Deng $\mathrm{G}$ and Qiu C: MicroRNA-188-5p promotes apoptosis and inhibits cell proliferation of breast cancer cells via the MAPK signaling pathway by targeting Rap2c. J Cell Physiol 235: 2389-2402, 2020.

36. Chen Y, Zheng Z, Wang J, Tang C, Khor S, Chen J, Chen X, Zhang Z, Tang Q, Wang C, et al: Berberine suppresses apoptosis and extracellular matrix (ECM) degradation in nucleus pulposus cells and ameliorates disc degeneration in a rodent model. Int J Biol Sci 14: 682-692, 2018.

37. Ruan Z, Ma H, Li J, Liu H, Jia H and Li F: The long non-coding RNA NEAT1 contributes to extracellular matrix degradation in degenerative human nucleus pulposus cells. Exp Biol Med (Maywood) 243: 595-600, 2018.

38. Zhang J, Li Z, Chen F, Liu H, Wang H, Li X, Liu X, Wang J and Zheng Z: TGF- $\beta 1$ suppresses CCL3/4 expression through the ERK signaling pathway and inhibits intervertebral disc degeneration and inflammation-related pain in a rat model. Exp Mol Med 49: e379, 2017.

39. Li S, Hua W, Wang K, Gao Y, Chen S, Liu W, Song Y, Wu X, Tu J, Kang L, et al: Autophagy attenuates compression-induced apoptosis of human nucleus pulposus cells via MEK/ERK/NRF1/Atg7 signaling pathways during intervertebral disc degeneration. Exp Cell Res 370: 87-97, 2018.

(i) $\Theta$ This work is licensed under a Creative Commons Attribution-NonCommercial-NoDerivatives 4.0 International (CC BY-NC-ND 4.0) License. 